\title{
Wake Properties of a Stripline Beam Kicker
}

\author{
B. R. Poole \\ G. J. Caporaso, W. C. Ng \\ This paper was prepared for submittal to the \\ 1997 Particle Accelerator Conference \\ Vancouver, B.C., Canada \\ May 12-16, 1997
}

May 27, 1997

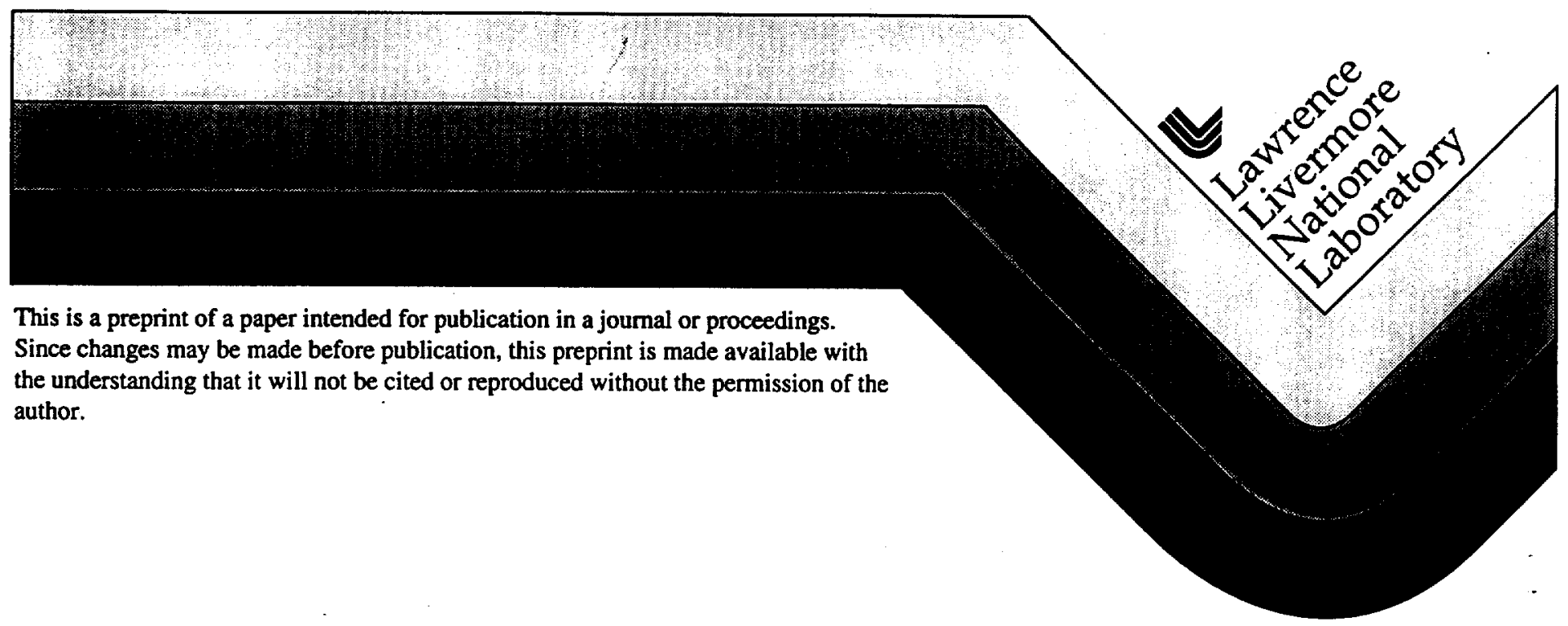




\section{DISCLAIMER}

This document was prepared as an account of work sponsored by an agency of the United States Government. Neither the United States Government nor the University of California nor any of their employees, makes any warranty, express or implied, or assumes any legal liability or responsibility for the accuracy, completeness, or usefulness of any information, apparatus, product, or process disclosed, or represents that its use would not infringe privately owned rights. Reference herein to any specific commercial product, process, or service by trade name, trademark, manufacturer, or otherwise, does not necessarily constitute or imply its endorsement, recommendation, or favoring by the United States Government or the University of California. The views and opinions of authors expressed herein do not necessarily state or reflect those of the United States Government or the University of California, and shall not be used for advertising or product endorsement purposes. 


\title{
WAKE PROPERTIES OF A STRIPLINE BEAM KICKER
}

\author{
Brian R. Poole, George J. Caporaso, and Wang C. Ng \\ Lawrence Livermore National Laboratory
}

\section{Abstract}

The transport of a high current relativistic electron beam in a stripline beam kicker is strongly dependent on the wake properties of the structure. The effect of the beam-induced fields on the steering of the beam must be determined for a prescribed trajectory within the structure. A 3-D time domain electromagnetic code is used to determine the wake fields and the resultant Lorentz force on the beam both for an ultra-relativistic electron beam moving parallel to the beamline axis as well as a beam that follows a curved trajectory through the structure. Usually in determining the wake properties of the structure, a wake impedance is found for a beam that is moving parallel to the beamline axis. However, we extend this concept to curved trajectories by calculating beam induced forces along the curved trajectory. Comparisons are made with simple transmission line models of the structure. The wake properties are used in models to transport the beam self-consistently through the structure.

\section{INTRODUCTION}

The stripline beam kicker is designed to spatially separate a high current electron beam for transport into two separate beamlines. Figure 1 shows a schematic representation of the geometry under consideration.

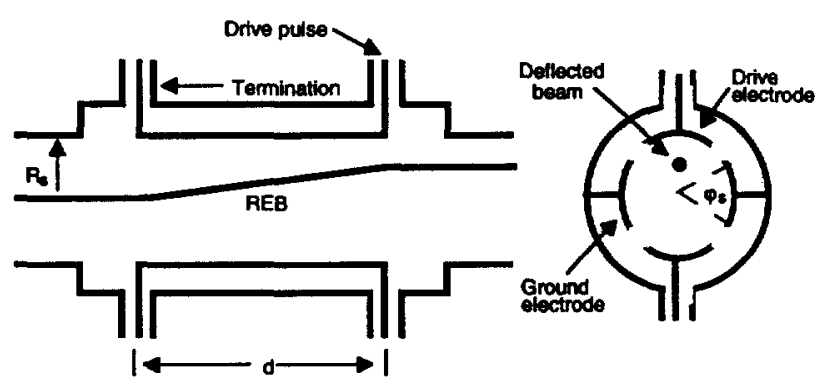

Figure 1. Kicker geometry and beam trajectory

An external pulse is applied to the downstream end of the kicker and the beam is deflected into one of two beamlines. The beam kicker is conceptually similar in design to a stripline beam position monitor.[1] However, the beam position monitor is typically used in high-energy accelerator applications with small beam current, to determine the transverse beam location by measurement of induced voltages on the striplines. In our application, the beam current is sufficiently large as to generate beaminduced voltages and currents on the strip transmission lines comparable with the externally applied voltages and currents. The dipole component of the beam-induced fields can contribute to the steering of the beam in addition to the externally applied steering fields. The wake impedance characterizes the strength of the interaction.

\section{ANALYSIS}

The wake impedance is a measure of the spectral characteristic of the integrated Lorentz force on a test charge, $q$ traveling a fixed distance $s$, behind a source charge, $Q$. The concept of the wake potential and transverse wake impedance of a structure are discussed by DeFord, [2] and are defined as

$$
\begin{gathered}
\mathbf{W}(s)=\left.\frac{1}{Q} \int_{-\infty}^{\infty} d z(\mathbf{E}+\mathbf{v} \times \mathbf{B})\right|_{t=(s+z) / v} \\
\mathbf{Z}_{t}(\omega)=\frac{j}{v} \int_{-\infty}^{\infty} \mathbf{W}_{t}(s) e^{j k s} d s
\end{gathered}
$$

where $\omega=k v$.

This type of stripline structure has been discussed in some detail by $\mathrm{Ng}$.[1] A simple model for the dipole wake impedance can be found using a simple transmission line model as shown in Figure 2.

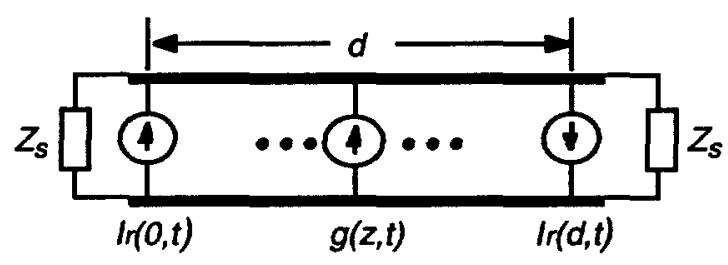

Figure 2. Transmission line model

To account for the curved trajectory of the beam within the structure, an equivalent shunt dipole current, $g(z, t)$ will be continuously setup along the transmission line to account for the change in the beam's dipole return current. The current sources at the ends of the structure represent the dipole gap return currents. Therefore, the transmission line equations representing this model are given by

$$
\frac{\partial V}{\partial z}=-L \frac{\partial I}{\partial t}
$$

and

$$
\frac{\partial I}{\partial z}=-C \frac{\partial V}{\partial t}+g(z, t)
$$


where

$$
g(z, t)=\frac{2}{\pi R_{s}} \sin \left(\frac{\phi_{s}}{2}\right) \frac{\partial}{\partial z}\left[r(z) I_{B}(t-d / c-z / c)\right]
$$

and $r(z)$ represents the trajectory of the beam through the structure.

If the beam is moving at a constant offset in the structure, the only dipole current sources in the transmission line model are the upstream and downstream dipole gap return currents. The wakes are then found by application of Equations (1) and (2) to the fields along the transmission line and analytic expressions for the transverse dipole ( $m=$ 1 ) impedance is found for a matched transmission line as

$$
\begin{aligned}
Z_{t}(\omega) & =\frac{8 c Z_{s}}{\pi^{2} R_{s}^{2}} \sin ^{2}\left(\frac{\phi_{s}}{2}\right)\left(\frac{1}{\omega}\right)\left[\sin ^{2}\left(\frac{\omega d}{c}\right)\right. \\
& \left.+j \sin \left(\frac{\omega d}{c}\right) \cos \left(\frac{\omega d}{c}\right)\right]
\end{aligned}
$$

The effect of $g(z, t)$ is to set up waves continuously along the transmission line which interact with the test charge as opposed to the case when the beam is moving parallel to the axis and the perturbed current is only generated at the gaps at the beginning and end of the structure. The continuous source distribution will significantly alter the wake properties of the structure.

For example, if the beam enters on axis and is steered off axis by an externally applied field as in the beam kicker we expect a reduction in the transverse dipole impedance since no beam return dipole current is present at the upstream gap. Also, the presence of the continuous distribution of sources will lead to a superposition of waves with differing phases to interact with the test charge which can lead to a reduction in the impedance as well as a redistribution of the spectral content of the impedance associated with the structure. $\mathrm{A}$ beam transport model based on the effect of the distributed shunt current sources is being developed.[3]

\section{MODELING}

We use a 3-D finite-difference time-domain (FDTD) code, TSAR (Temporal Scattering and Response) to model the wake properties of the structure and compare the results with the simple transmission line model for the structure as described by Ng.[1] The code uses a 3-D grid composed of cubical cells for the FDTD analysis, with each cell being assigned a given material property. The cell size is typically chosen to resolve the shortest wavelength expected in the problem and is chosen small enough to accurately represent the smallest dimension in the model. In addition, to satisfy stability requirements for the FDTD field solver, the Courant condition for the time step must be satisfied. This condition is $(d t<d x / 2 c)$ where $d x$ is the length of a cell edge. It is also important to resolve the lowest frequency that the electron beam may excite, which determines the total number of time steps to be run for a specific problem.

In our numerical calculations, the length of the structure was $10 \mathrm{~cm}$, the strip radius was $1.65 \mathrm{~cm}$, the subtended angle of each of the 4 strips was 55 degrees and the enclosure radius was $1.9 \mathrm{~cm}$. The structure was terminated with $25 \Omega$ resistors which were chosen based on a frequency domain calculation of the TEM impedance of the structure for our geometry. Even to represent the gross geometric characteristics of the structure using cubical cells requires a cell size of 0.5 $\mathrm{mm}$ making the computational size of this problem $8 \times 10^{6}$ cells and requires a time step of $.83 \mathrm{ps}$ as dictated by the Courant condition. The axial ends of the problem space had MUR radiation boundary conditions imposed to prevent undesired reflections. The MUR boundary conditions were located $25 \mathrm{~cm}$ away from each end of the structure to allow a sufficient decay of evanescent waves. The problem was run for 16000 time steps.

For modeling purposes the trajectory of the beam was either a constant offset beam at a radius of 1 $\mathrm{cm}$ or a trajectory of the form

$$
r(z)=\frac{r_{0}}{2}\left[1-\cos \left(\frac{\pi z}{d}\right)\right]
$$

where $d$ is the length of the structure. In this case, the beam enters the structure on axis and leaves at an offset of $r_{0}=1 \mathrm{~cm}$. While this trajectory is somewhat artificial, it was required to have the beam enter and leave the problem space normal to the plane of the MUR radiation boundary conditions at the axial ends of the problem space for stability of the code. Figures $3 a$ and $3 \mathrm{~b}$ show comparisons of the analytic model of the offset beam with results obtained numerically through the modeling code for the transverse dipole impedance for a beam with a constant offset. As is seen, there is excellent low frequency agreement with the numerical results for the constant offset beam with the analytic dipole impedance. Figures $4 a$ and $4 b$ show the results due to the distributed source $g(z, t)$ arising from the trajectory defined in equation 7 . Note that these plots do not include the contributions from the dipole gap currents. Again, there is excellent low frequency agreement between the distributed source model and the code results. These results must be subtracted from the constant offset impedance results to obtain the total curved trajectory dipole impedances. The effect of the curved trajectory, as expected was to reduce the impedance and redistribute the impedance spectra. The deviations at the higher frequencies may be due to the high frequency impedance of the wire resistors used in the FDTD code which are not included in the analytic model since we are assuming the striplines to be matched. 


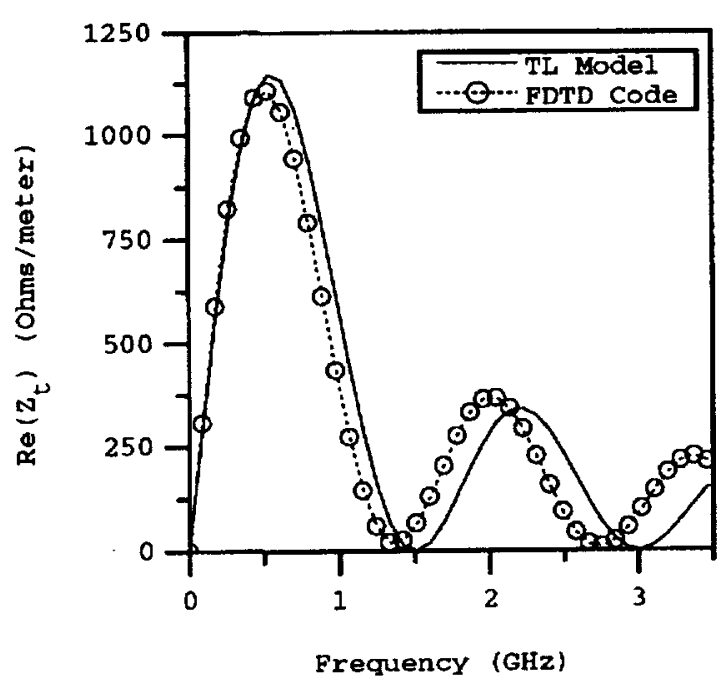

Figure 3a. Real part of impedance for offset beam

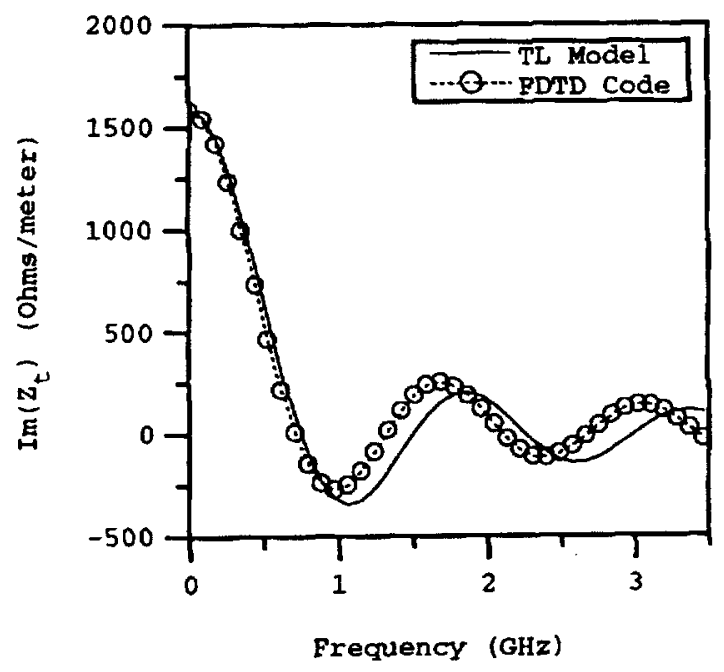

Figure 3b. Imaginary part of impedance for offset beam

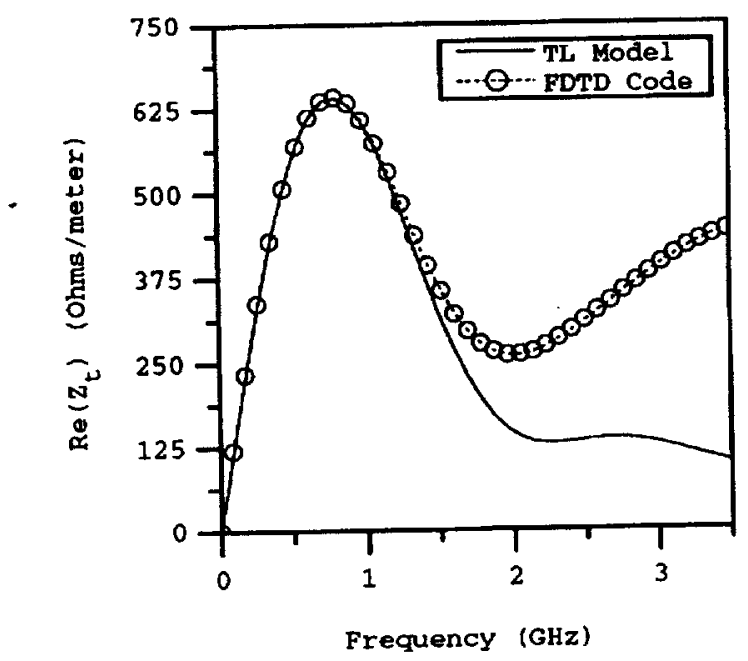

Figure 4a. Contribution to real part of dipole impedance for curved trajectory from distributed source term, $g(z, t)$

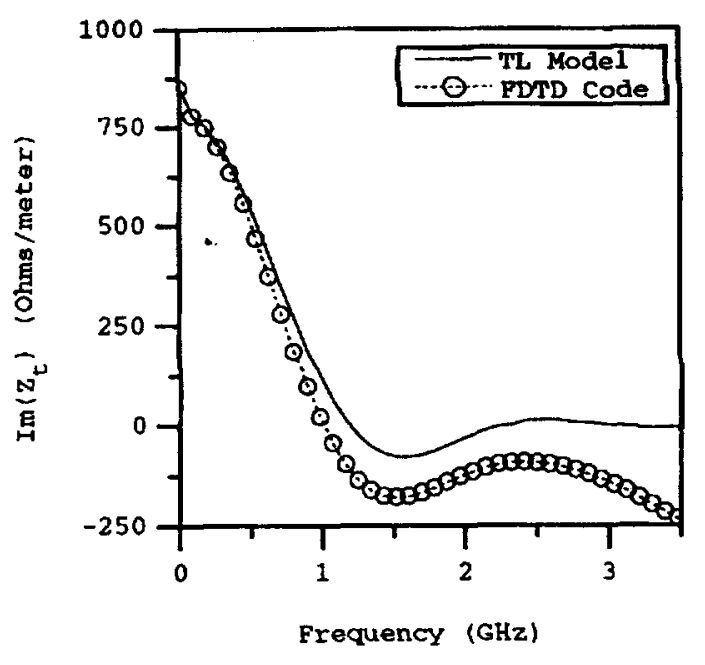

Figure $4 \mathrm{~b}$. Contribution to imaginary part of dipole impedance for curved trajectory from distributed source term, $g(z, t)$

\section{CONCLUSION}

The effect of including more complex beam trajectories in wake impedance calculations can have significant impact on the nature of the beam interaction. These effects are particularly relevant in the design of highcurrent beam transport systems where the beam induced fields may be large. It is found that the wake impedance spectra is altered due to the introduction of a continuous distribution of shunt transmission line currents to account for the change in dipole return current as the beam is deflected in the structure. The resultant superposition of waves generated by the distributed current sources is to redistribute the spectral content of the wake impedance. We see from our modeling the wake impedance at very low frequency is reduced by a factor of 2 indicating the effect of beam induced steering is not as significant as in the beam offset case.

\section{ACKNOWLEDGMENT}

Stimulating discussions with S. Nelson, C. Shang, and Yu Ju Chen of Lawrence Livermore National Laboratory are gratefully acknowledged. This work was performed under the auspices of the U.S. Department of Energy by the Lawrence Livermore National Laboratory under contract number W-7405-ENG-48.

\section{REFERENCES}

[1] K.-Y. Ng, Particle Accelerators, 23, 93-102 (1988).

[2] J. F. DeFord, G. D. Craig Particle Accelerators, 37-38, 111121 (1992).

[3] G. J. Caporaso, Yu Ju Chen, B. R. Poole, "Transmission Line Analysis of Beam Deflection in a BPM Stripline Kicker," these proceedings. 


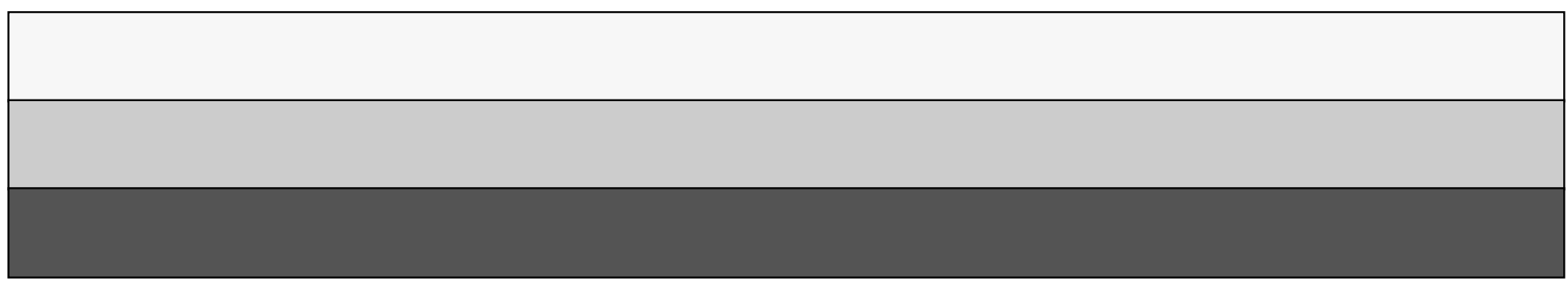

